\title{
A Single Administration of Riluzole Applied Acutely After Spinal Cord Injury Attenuates Pro- Inflammatory Activity And Improves Long-Term Functional Recovery In Rats
}

\section{Qichao Wu}

Beijing Chao-Yang Hospital: Beijing Chaoyang Hospital

Wenkai Zhang

Beijing Luhe Hospital

Shuo Yuan

Beijing Chao-Yang Hospital: Beijing Chaoyang Hospital

Yanjun Zhang

Beijing Luhe Hospital

Wenxiu Zhang

Beijing Luhe Hospital

Yan Zhang

Beijing Luhe Hospital

Xueming Chen

Beijing Luhe Hospital

Lei Zang ( $\nabla$ zanglei@ccmu.edu.cn )

Beijing Chaoyang Hospital https://orcid.org/0000-0003-1403-4159

\section{Research Article}

Keywords: microglia/macrophage, neuroinflammation, riluzole, spinal cord injury, ST2

Posted Date: July 30th, 2021

DOI: https://doi.org/10.21203/rs.3.rs-662233/v1

License: (c) (i) This work is licensed under a Creative Commons Attribution 4.0 International License. Read Full License

Version of Record: A version of this preprint was published at Journal of Molecular Neuroscience on January 6th, 2022. See the published version at https://doi.org/10.1007/s12031-021-01947-y. 


\section{Abstract}

After spinal cord injury $(\mathrm{SCl})$, emergency treatment intervention can minimize tissue damage, which is closely related to the recovery of long-term function. Here, we examined whether the administration of a single dose of riluzole $(6 \mathrm{mg} / \mathrm{kg})$ immediately after $\mathrm{SCl}$ was a critical window for the drug to exert its regulatory effect and limit long-term neurological deficits. The animals were sacrificed 1 day after administration for investigation of neuronal survival and a potential neuroinflammatory response, and sacrificed in the 6th week for assessment of neurological function. Riluzole applied in a single dose immediately post-SCl decreased the mRNA level of interleukin-1 $\beta$ at $6 \mathrm{~h}$, reduced the destruction of neurons, and reduced the activation of microglia/macrophage M1 expression at day 1 post-SCI. Additionally, riluzole-treated rats showed higher expressions of interleukin-33 and its receptor ST2 in microglia/macrophages of the spinal cord than vehicle-treated rats, suggesting that this signaling pathway might be involved in microglia/macrophage-mediated inflammation. At 6 weeks, riluzole-treated rats exhibited higher motor function scores than vehicle-treated controls. In addition, riluzole-treated rats exhibited higher expression of GAP43 protein and shorter N1 peak latency and larger N1-P1 amplitude in motor-evoked potentials, compared to vehicle-treated rats. Together, these data suggested that early application of riluzole after $\mathrm{SCl}$ could be crucial for long-term functional recovery, so it may represent a promising therapeutic candidate within the critical therapeutic window for acute SCl.

\section{Headings}

1. Riluzole immediately applied in a single dose post-spinal cord injury (SCl), decreased the mRNA level of interleukin (IL)-1 $\beta$ at $6 \mathrm{~h}$, and reduced the destruction of neurons at day 1 post-SCl.

2. At 6 weeks, riluzole-treated rats exhibited better neurological function compared to vehicle-treated rats. This advantage may have been due to riluzole intervention during the acute phase of spinal cord injury.

3. Anti-inflammatory effects may be another important mechanism of riluzole, in addition to its welldescribed function of reducing excitotoxicity by blocking glutamatergic neurotransmission.

\section{Introduction}

Spinal cord injury (SCl) often causes functional disturbances in the sensory, motor, and autonomic nervous systems, and has become a social burden, with an the increased global incidence of SCl in recent years (Hall et al. 2019; James 2019). Secondary injury after SCl includes cell apoptosis, axonal degeneration, demyelination, and immune responses that are inseparable from the severity of neurological deficits (Fan et al. 2019). Although no effective therapy is available for this debilitating disorder, anti-inflammatory therapies have been suggested to reduce the neurological deficits caused by secondary injury after SCl (Kobashi et al. 2020). Recent studies suggested that early modulation of the microglia/macrophage-mediated inflammatory response could be crucial for the functional outcome (Bimbova et al. 2018; Lin et al. 2021). 
Microglia/macrophages play a central role in immune defense in the central nervous system (CNS) (Saijo et al. 2011; Kobashi et al. 2020). After SCl, microglia/macrophages are recruited to the injury site and produce pronounced inflammatory responses. Intriguingly, the mobilized microglia/macrophages exist in different functional phenotypes that play different roles in SCl (Kigerl et al. 2009). The "classic" M1 and "alternative" M2 phenotypes are the most studied and represent two generally opposite functions. M1 microglia/macrophages exert pro-inflammatory effects by recruiting inflammatory cells that produce cytokines such as interleukin (IL)-1 $\beta$, tumor necrosis factor (TNF)- $a$, and IL- 6 (Cherry et al. 2014; Zhang et al. 2019). Conversely, M2 microglia/macrophages are associated mainly with anti-inflammatory effects through the expression of transforming growth factor (TGF)- $\beta$, IL-10, and IL-13 (Cherry et al. 2014; Zhang et al. 2019). Emerging evidence suggests that inhibiting inflammation by modulating spinal microglia/macrophage polarization may have neuroprotective effects. For example, baicalein (Lai et al. 2018), curcumin (Lee et al. 2019), fumaric acid (Cordaro et al. 2017), minocycline (Afshary et al. 2020), and melatonin (Zhang et al. 2019) have been shown to protect neuronal tissues from SCl by modulating microglia/macrophage polarization. Some physical therapies such as aerobic exercise (Donia et al. 2019) and hypothermia (Chevin et al. 2018) are also beneficial for SCl, possibly through similar mechanisms. Therefore, modulation of the microglia/macrophage-mediated inflammatory response represents a promising strategy to protect against tissue damage in the acute phase of $\mathrm{SCl}$.

One drug suggested for use in patients with $\mathrm{SCl}$ is riluzole, which was approved by the U.S. Food and Drug Administration for treatment of amyotrophic lateral sclerosis. Recent studies showed that riluzole can be used not only to treat chronic neurodegenerative disorders, including Alzheimer disease and Parkinson's disease, but also to limit CNS injury from cerebral ischemia and SCI (Nagoshi et al. 2015; Liu et al. 2018). Although riluzole has entered phase IIB/III trials for SCI (Fehlings et al. 2016), its mechanism of action is only partially understood. The classic mechanism of riluzole involves alleviating glutamate toxicity, which contributes to the secondary injury after SCl. Although increasing evidence shows that riluzole also exerts neuroprotection through anti-inflammatory effects (Karadimas et al. 2015; Jiang et al. 2016), our study focused on its application in the critical first moments after SCl, and verified whether the administration of a single dose of riluzole at this stage minimized secondary injury. The results showed that riluzole immediately applied in a single dose post-SCl suppressed the microglia/macrophagemediated inflammatory response, reduced the destruction of neurons, and improved long-term neurological function. These results suggested that riluzole may represent a promising early therapeutic candidate to treat SCI.

\section{Materials And Methods}

\section{Animals and surgery for SCl}

All procedures were implemented in accordance with the Institutional Animal Care and Use Committee of Capital Medical University (Beijing, China). A total of 109 adult female Wistar rats (9-weeks-old, 220-240 g) were purchased for studies from the Vital River Laboratory Animal Technology Co., Ltd (Beijing, China), including seven rats that were excluded from further assessments due to death. In the first stage of our 
study, 48 rats were used to detect the expressions of IL-1 $\beta$ at $0,6,12$, and $24 \mathrm{~h}$ after SCl, which were divided into two groups at each time point: $\mathrm{SCl}+$ Vehicle and $\mathrm{SCl}+$ Riluzole $(n=6 / g r o u p)$. In the second stage, 54 rats were randomly divided into three groups: Sham, $\mathrm{SCl}+$ Vehicle, and $\mathrm{SCl}+$ Riluzole $(\mathrm{n}=$ 18/group) for the detection of neuronal apoptosis and long-term motor function in SCl rats.

Rats were secured to the table after being anesthetized with a mixture of oxygen and isoflurane (1.5$2.0 \%)$. Fur over the surgical area was shaved and the skin disinfected. A dorsal midline incision was made at the T6 to T12 levels, and the skin, subcutaneous tissue, and fascia were cut. The spinous processes were exposed by blunt dissection of the underlying muscle layers. The thoracic vertebrae at T9, $\mathrm{T} 10$, and T11 were excised to expose the spinal cord. The SCI model was generated by allowing a rod (height: 25 mm; weight: 10 g; diameter: 3 mm, Impactor Model III; Rutgers University, New Brunswick, NJ, USA) to fall onto the spinal cord. After injury, muscles and skin were sutured under sterile conditions. SCl rats were administered an intraperitoneal (i.p.) injection of $6 \mathrm{mg} / \mathrm{kg}$ riluzole or the same volume of vehicle immediately after surgery. Finally, the rats were kept warm with an electric blanket until they had fully recovered from the anesthesia.

\section{Animal care}

The rats were housed at a constant ambient temperature with a $12 \mathrm{~h}$ light-dark cycle and provided with food and water ad libitum. SCl rats were artificially urinated four times a day to prevent urinary tract infections and interventional bladder filling. The hind limbs of the rats were washed with saline, and the skin was dried with a hair dryer.

\section{Locomotion assay}

The Basso, Beattie, Bresnahan (BBB) (Basso et al. 1995) locomotor rating scale and inclined plane test (Rivlin et al. 1977) were used to evaluate the functional recovery of rats on postoperative days $1,3,7,14$, $21,28,35$, and 42 . The BBB score is based on the animal's foot placement, limb movement, gait, and coordination. A score of 21 is normal. For the evaluation of hind limb strength, we performed the inclined plane test. Animals were placed on an inclined plane, which was then angled until they could no longer maintain their footing. The time and angle were recorded. The test was repeated three times for each rat, and data were averaged for analysis.

\section{Electrophysiological evaluation}

Motor evoked potentials (MEPs) were recorded to evaluate the recovery of the motor systems after SCl. The MEPs test assay was performed as previously described (Xu et al. 2017). Briefly, rats were anesthetized with gas mixtures of oxygen and isoflurane. Stimulator electrodes were inserted subcutaneously above the anterior fontanelle and the active recording electrodes were placed in each Achilles' tendon for measurement of MEPs. The N1-P1 amplitude and N1 peak latency were used to analyze the effect of neurological recovery.

\section{Nissl straining}


On day 1 after surgery, rats were euthanized with a mixture of oxygen and isoflurane (1.5-2.0\%), perfused with saline, and fixed with $4 \%$ paraformaldehyde. The spinal cord was then removed, fixed in $4 \%$ paraformaldehyde solution, and dehydrated in $30 \%$ sucrose for 3 days before being stored at $4^{\circ} \mathrm{C}$. Frozen spinal cords were cut into $20 \mu \mathrm{m}$ sections with a microtome (CM1950; Leica, Wetzlar, Germany). According to the manufacturer's instructions, we stained the sections with Nissl stain. Finally, we acquired brightfield images by light microscopy (Nikon, Tokyo, Japan).

\section{Immunofluorescence}

Frozen sections $(20 \mathrm{~mm})$ were permeabilized three times with phosphate-buffered saline/Tween 20 (PBST) for $15 \mathrm{~min}$, blocked with 10\% goat serum for $30 \mathrm{~min}$, and incubated overnight with primary antibody to NeuN (ab104224, 1:200: Abcam, Cambridge, UK), cleaved caspase-3 (ab49822, 1:200; Abcam), Iba I (01919741, 01226723, 1:100; Wako, Osaka, Japan), ST2 (ab25877, 1:100; Abcam), CD16 (ab109223, 1:100; Abcam), or CD206 (sc-58986, 1:200; Santa Cruz Biotechnology, Santa Cruz, CA, USA). Subsequently, the sections were washed in PBST for $30 \mathrm{~min}$ and incubated with secondary antibody (A32723, A32754, 1:100; Invitrogen, Carlsbad, CA, USA) for $8 \mathrm{~h}$. Finally, sections were washed in PBST for $30 \mathrm{~min}$ and then incubated with 4',6-diamidino-2-phenylindole for $30 \mathrm{~s}$. The stained sections were analyzed in fields/sections with a confocal laser scanning microscope (Nikon).

\section{Western blot analysis}

Protein expressions of GAP-43 (8945, 1:1,000; Cell Signaling Technology, Danvers, MA, USA), ST2 (ab25877, 1:2,000; Abcam), IL-33 (ab207737, 1:2,000; Abcam), CD16 (ab109223, 1:2,000; Abcam), and CD206 (sc-58986, 1:1,000; Santa Cruz Biotechnology) were determined in spinal cord homogenates by western blot analysis as previously described ${ }^{[25]}$. After the protein concentration was measured using bovine serum albumin, the proteins were separated by SDS-PAGE and then transferred onto a polyvinylidene difluoride membrane. Next, the membranes were blocked with $5 \%$ nonfat milk for 60 min and incubated with primary antibodies for $12 \mathrm{~h}$ at $4^{\circ} \mathrm{C}$. Finally, the membranes were incubated with secondary antibody (A32723, A32754, 1:5,000; Invitrogen) for $1 \mathrm{~h}$, and images were acquired (BioSpectrum 515; LabMode, Borehamwood, UK). Image J (National Institutes of Health, Bethesda, MD, USA) was used to visualize the reaction products for quantifying protein expression.

\section{Real-time PCR}

Total RNA was extracted from spinal cord tissue with TRIzol reagent (Invitrogen), and cDNA was synthesized in accordance with the instructions (Tiangen Biotech, Beijing, China). We performed real-time PCR on a quantitative PCR system (Applied Biosystems 7500 Real-Time PCR System; Thermo Fisher Scientific, Waltham, MA, USA) under the following conditions: $95^{\circ} \mathrm{C}$ for $5 \mathrm{~min}$ followed by 40 cycles of $95^{\circ} \mathrm{C}$ for $15 \mathrm{~s}$ and $60^{\circ} \mathrm{C}$ for $30 \mathrm{~s}$. The relative expression levels of the target genes were normalized to that of the housekeeping gene, glyceraldehyde 3-phosphate dehydrogenase. The expression levels of ST2, IL33, CD16, CD32, CD86, CD206, iNOS, Arg1, IL-4, and TGF- $\beta$ were calculated using the $2^{-\Delta \Delta C T}$ method. The primer series are listed in Table 1. 


\begin{tabular}{|c|c|c|}
\hline Genes & & Primers (5'-3') \\
\hline \multirow[t]{2}{*}{ ST2 } & Forward & ATGATTGGCAAATGGAGAAT \\
\hline & Reverse & TTCTAGACCCCAGGATGTTT \\
\hline \multirow[t]{2}{*}{ IL-33 } & Forward & GTGCAGGAAAGGAAGACTCG \\
\hline & Reverse & TGGCCTCACCATAAGAAAGG \\
\hline \multirow[t]{2}{*}{ CD16 } & Forward & GCATGCTACTTACGGTTTCC \\
\hline & Reverse & TGCGAGATGAGGCTTTTGT \\
\hline \multirow[t]{2}{*}{ CD32 } & Forward & AGCTGCTGTCGCTGGAAT \\
\hline & Reverse & GGATGCTTGAGAAGTGAATAGG \\
\hline \multirow[t]{2}{*}{ CD86 } & Forward & TAGGGATAACCAGGCTCTAC \\
\hline & Reverse & CGTGGGTGTCTTTTGCTGTA \\
\hline \multirow[t]{2}{*}{ CD206 } & Forward & AGCTGCTGTCGCTGGAAT \\
\hline & Reverse & GGATGCTTGAGAAGTGAATAGG \\
\hline \multirow[t]{2}{*}{ iNOS } & Forward & CAGCATCCACGCCAAGAA \\
\hline & Reverse & САACTCGCTCCAAGATCCCT \\
\hline \multirow[t]{2}{*}{ Arg1 } & Forward & TGTGGGAAAAGCCAATGAAC \\
\hline & Reverse & GGTGTCAGCGGAGTGTTG \\
\hline \multirow[t]{2}{*}{ IL-4 } & Forward & ACAGGAGAAGGGACGCCAT \\
\hline & Reverse & GAAGCCCTACAGACGAGCTCA \\
\hline \multirow[t]{2}{*}{ TGF- $\beta$} & Forward & AGGCGGTGCTCGCTTTGTA \\
\hline & Reverse & ATTGCGTTGTTGCGGTCC \\
\hline \multirow[t]{2}{*}{ GAPDH } & Forward & GCAAGTTCAACGGCACAG \\
\hline & Reverse & GCCAGTAGACTCCACGACAT \\
\hline
\end{tabular}

\section{Statistical analysis}

All data were analyzed using Prism software, version 8.4.2 (GraphPad, San Diego, CA, USA). We used Student's $t$-test to determine the statistical significance of the differences between $\mathrm{SCl}+$ vehicle, and $\mathrm{SCl}$ + riluzole. One-way analysis of variance and Tukey's post hoc analysis were used to compare data between multiple groups. Two-way analysis of variance was used to analyze the difference in mRNA 
expression levels of IL-1 $\beta$ between $\mathrm{SCl}+$ vehicle and $\mathrm{SCl}+$ riluzole. Numerical data are shown as the mean $\pm \mathrm{SEM}$, and $\mathrm{P}<0.05$ was considered statistically significant.

\section{Results}

\section{Riluzole reduces the release of the pro-inflammatory cytokine II-1 $\beta$ after $\mathrm{SCl}$ (short-term results).}

The activation of a neuroinflammatory response after $\mathrm{SCl}$ led to inflammatory mediators and inflammatory cells aggregating and infiltrating the injury center of spinal cord tissue. We tested whether a single dose of riluzole ( $6 \mathrm{mg} / \mathrm{kg}$; i.p.) decreased the expression of the pro-inflammatory cytokine IL-1 $\beta$ after $\mathrm{SCl}$. Figure $1 \mathrm{~b}$ shows a sharp increase of mRNA levels of IL-1 $\beta$ around the injury center at $6 \mathrm{~h}$ after $\mathrm{SCl}$ in the $\mathrm{SCl}+$ vehicle group, which remained 8.5 -fold higher than that in naive spinal cord, followed by a rapid decrease within $24 \mathrm{~h}$. Riluzole-treated rats exhibited lower mRNA levels of IL-1 $\beta$ in the injured spinal segment at $6 \mathrm{~h}$ after $\mathrm{SCl}$, but remained 4.4 -fold higher than that in the naive spinal.

\section{Riluzole increases the expressions of IL-33 and ST2 in microglia/macrophages of the spinal cord after $\mathrm{SCl}$ (short-term results).}

Immunostaining showed a higher percentage of double-labeled ST2 ${ }^{+} / \mathrm{Iba}^{+}$cells in riluzole-treated SCl rats than in vehicle-treated $\mathrm{SCl}$ rats $(\mathrm{P}<0.01 ; \mathrm{Fig} .2 \mathrm{a}, \mathrm{f})$. Similarly, we found that $\mathrm{SCl}$ rats exhibited significantly increased mRNA and protein levels of both IL-33 and ST2, as compared with levels in vehicle-treated rats (Fig. $2 b-e, g$ ). Collectively, these findings suggested that riluzole increased the expression of IL-33 and ST2 in spinal cord microglia/macrophages of SCl rats.

\section{Riluzole inhibits microglia/macrophage M1 expression in SCI rats (short-term results).}

Compared with the vehicle-treated group, riluzole-treated rats showed lower mRNA levels of the M1-like markers, CD16, CD32, CD86, and iNOS, and a lower level of CD16 protein on day 1 post-SCl (Fig. 3c-e). Immunostaining showed that CD16 immunoreactivity was decreased $(P<0.01$, Fig. 3a, d) in the spinal cord of riluzole-treated rats, when compared with that in vehicle-treated rats. However, there was no statistical difference in the mRNA levels of M2-like markers (CD16, CD32, CD86, and iNOS) (Fig. 3e) and the protein levels and immunoreactivities of CD206 between the riluzole-treated and vehicle-treated groups (Fig. 3b-d).

\section{Riluzole reduces neuronal loss after acute $\mathrm{SCl}$ (short-term results).}

Nissl staining showed that the percentage of surviving neurons was higher in the spinal cord ventral horn of riluzole-treated rats at day 1 post-SCI than in that of vehicle-treated rats $(P<0.01$; Fig. $4 a, d)$.

Furthermore, we performed double-immunofluorescence staining for cleaved-caspase-3 and NeuN (Fig. 4b). Compared with the vehicle-treated group, riluzole-treated rats showed a lower percentage of cleaved-caspase-3 and NeuN double-labeled neurons ( $P<0.01$, Fig. $2 e)$ in the spinal cord at day 1 post$\mathrm{SCl}$, suggesting less neuronal apoptosis. 


\section{Riluzole promotes recovery of neurological function after SCl (long-term results).}

Riluzole improved functional restoration after $\mathrm{SCl}$, as indicated by higher scores in the inclined plane test and BBB assay $(P<0.001$, Fig. 5h, i), suggesting that riluzole may improve long-term recovery of locomotion function after SCI. Western blot analysis showed that GAP43 expression was significantly higher in the riluzole-treated group than in the vehicle-treated group $(P<0.001, F i g .5 g)$. Furthermore, riluzole-treated SCI rats showed a shorter N1 peak latency (the first negative deflection) and longer N1-P1 amplitude compared to vehicle-treated rats $(P<0.001$, Fig. 5b, $c)$. These findings suggested that riluzole may promote recovery of neurological function after SCl.

\section{Discussion}

Neuroinflammation is inseparable from the severity of neurological deficits after SCI. IL-1 $\beta$, a key proinflammatory factor in the CNS, is released only $2 \mathrm{~h}$ after SCl. It promotes a large number of neutrophils to enter the CNS and the production and release of neurotoxic substances such as excitatory amino acids and free radicals, and triggers calcium ion overload, all of which are considered to be the key factors in the destruction of spinal cord tissue. Our results showed that application of a single dose of riluzole in the critical first moments after SCI reduced the mRNA level of IL-1 $\beta$ in the injured spinal segment at $6 \mathrm{~h}$ after $\mathrm{SCl}$, and improved long-term neurological function. These results showed that early application of riluzole after $\mathrm{SCl}$ could be crucial for the long-term functional recovery and it may represent a promising early therapeutic candidate for SCl.

Riluzole is a sodium channel blocker with the advantage of being able to pass through the blood-brain barrier; and it has been shown to have therapeutic efficacy in multiple chronic neurodegenerative disorders, as well as acute CNS injuries (Nagoshi et al. 2015; Liu et al. 2018). Scientists have characterized the pharmacological mechanism of riluzole for more than 20 years, showing its neuroprotective effect was mainly achieved by inhibiting the excitatory neurotoxicity of glutamate (Nagoshi et al. 2015). In recent years, emerging evidence suggested that riluzole also inhibited inflammatory responses after SCl, and improved neurological function (Nagoshi et al. 2015). In the present study, we also found that riluzole reduced the mRNA level of IL-1 $\beta$ after SCl, and that this effect may be associated with IL-33/ST2-dependent microglial/macrophage infiltration. Our study suggests that anti-inflammatory effects may be another important mechanism of riluzole, in addition to its welldescribed function of reducing excitotoxicity by blocking glutamatergic neurotransmission.

$\mathrm{SCl}$ often triggers widespread inflammation, which further leads to neuropathy accompanied by limited functional restoration (Oyinbo 2011; Mortazavi et al. 2015). Recent studies have highlighted the aggregation of pro-inflammatory mediators including, IL-1 3 , IL-6, and TNFa, which may interact with neuroglia, especially microglia, augmenting secondary injury and eventually leading to neurological deficits (Bartholdi et al. 1997; Allison et al. 2015). Microglia are thought to have a central role in the immune response to SCI (Saijo et al. 2011; Kobashi et al. 2020). The M1 microglial phenotype generally exerts pro-inflammatory effects by recruiting inflammatory cells that produce cytokines and cause further 
neurological deficits (Cherry et al. 2014; Zhang et al. 2019). Conversely, M2 microglia are mainly associated with anti-inflammatory, neuroprotective effects (Cherry et al. 2014; Zhang et al. 2019). Our previous studies have shown that riluzole reduced the expression of M1 and increased the expression of M2 on the 7th day after SCl (Wu et al. 2020). In the present study, on the first day after SCl, we found that riluzole reduced the expression of $\mathrm{M} 1$, but did not increase the expression of $M 2$. The above differences may be due to the different activation peaks of M1 and M2 microglia. In the 3-10 days after SCl, M2-type microglia gradually occupy the dominant position, phagocytize and clear the myelin sheath, promote the generation of new myelin sheath, and provide a more suitable microenvironment for neurons, so that the nervous system can be effectively protected (Gensel et al. 2015). However, M1 type microglia can be activated in the early stage of CNS injury, and produce a large number of inflammatory factors, causing damage to tissues (Gensel et al. 2015). Therefore, the difference in the expression of M1 microglia was detected on the first day after SCl. Riluzole-treated rats showed better long-term neurological function recovery, which was closely related to early reduction of M1 microglia expression, to reduce damage to neural tissue.

Although the regulation mechanism of microglia/macrophage-mediated inflammatory response in the nervous system remains unclear, several critical signaling pathways may be involved. For example, lentivirus-mediated downregulation of a-synuclein promotes polarization toward an M2 phenotype, conferring immune defense in SCl rats (Zeng et al. 2019). In addition, curcumin may inhibit STAT1 and STAT3 activation and improve functional restoration after ischemic stroke (Qin et al. 2012; Liu et al. 2017). In our study, riluzole-treated SCI rats exhibited higher mRNA and protein levels of IL-33 and ST2 than did vehicle-treated SCI rats. Immunofluorescence showed that in the spinal cord, riluzole-treated SCI rats had a higher percentage of double-labeled ST2+/lba I + cells than did vehicle-treated rats. These data indicated that ST2 receptor expression in microglia/macrophage was altered after riluzole intervention. We therefore speculate that riluzole-mediated modulation of microglia/macrophage may be associated with changes in expression of IL-33/ST2 in the spinal cord after SCI.

In summary, our study focused on the application of riluzole in the critical first moments after $\mathrm{SCl}$. The results indicated that early application of riluzole after $\mathrm{SCl}$ suppressed the microglia/macrophagemediated inflammatory response and improved long-term neurological function. Thus, riluzole may represent a promising therapeutic candidate within the critical therapeutic window for acute SCl.

\section{Abbreviations}

$\mathrm{SCl}$, spinal cord injury; CNS, central nervous system; M1, M1 microglia/macrophage; M2, M2 microglia/macrophage; HE, hematoxylin and eosin; BBB, Basso, Beattie, Bresnahan; MEPs, Motor evoked potentials. IL, interleukin; TNF, tumor necrosis factor. TGF, transforming growth factor.

\section{Declarations}

\section{Funding}


This work was supported by the National Natural Science Foundation of China (81901241).

\section{Conflicts of interest}

All authors declare that there are no conflicts of interest.

\section{Availability of data and material}

The data that supported the findings of the present study are available from the corresponding authors upon reasonable request.

\section{Code availability}

Not applicable

Authors' contributions Designed research: L.Z., X.C., and Q.W. Performed research: Q.W., Y.Z., W.Z., and S.Y. Analyzed data: Q.W. and W.Z. Wrote the manuscript: Q.W., X.C., and Y.Z.

Ethics approval All animal experiments were approved by the Institutional Animal Care and Use Committee of Capital Medical University. The animals were handled according to the guidelines set forth by the Chinese National Institutes of Health.

\section{Consent to participate}

Not applicable, because this study did not involve human participants.

\section{Consent for publication}

Not applicable, because this study did not involve human participants.

\section{Acknowledgements}

Not applicable

\section{References}

1. Afshary K, Chamanara M, Talari B, Rezaei P, Nassireslami E (2020) Therapeutic Effects of Minocycline Pretreatment in the Locomotor and Sensory Complications of Spinal Cord Injury in an Animal Model. J MOL NEUROSCI 70:1064-1072. https://doi: 10.1007/s12031-020-01509-8

2. Allison DJ, Ditor DS (2015) Immune dysfunction and chronic inflammation following spinal cord injury. SPINAL CORD 53:14-18. doi 10.1038/sc.2014.184.

3. Bartholdi D, Schwab ME (1997) Expression of pro-inflammatory cytokine and chemokine mRNA upon experimental spinal cord injury in mouse: an in situ hybridization study. EUR J NEUROSCI 9:14221438. doi 10.1111/j.1460-9568. 
4. Basso DM, Beattie MS, Bresnahan JC (1995) A sensitive and reliable locomotor rating scale for open field testing in rats. J Neurotrauma 12:1-21. doi 10.1089/neu.

5. Bimbova K, Bacova M, Kisucka A, Pavel J, Galik J, Zavacky P, Marsala M, Stropkovska A, Fedorova J, Papcunova S, Jachova J, Lukacova N (2018) A Single Dose of Atorvastatin Applied Acutely after Spinal Cord Injury Suppresses Inflammation, Apoptosis, and Promotes Axon Outgrowth, Which Might Be Essential for Favorable Functional Outcome. INT J MOL SCI 19, 1106. https://doi: 10.3390/ijms19041106

6. Cherry JD, Olschowka JA, O'Banion MK (2014) Neuroinflammation and M2 microglia: the good, the bad, and the inflamed. J Neuroinflammation 11:98. doi 10.1186/1742-2094-11-98.

7. Chevin M, Guiraut C, Sebire G (2018) Effect of hypothermia on interleukin-1 receptor antagonist pharmacodynamics in inflammatory-sensitized hypoxic-ischemic encephalopathy of term newborns. J Neuroinflammation 15:214. doi 10.1186/s12974-018-1258-6.

8. Cordaro M, Casili G, Paterniti I, Cuzzocrea S, Esposito E (2017) Fumaric Acid Esters Attenuate Secondary Degeneration after Spinal Cord Injury. J NEUROTRAUM 34:3027-3040. doi 10.1089/neu.2016.4678.

9. Donia SA, Allison DJ, Gammage KL, Ditor DS (2019) The effects of acute aerobic exercise on mood and inflammation in individuals with multiple sclerosis and incomplete spinal cord injury. NEUROREHABILITATION 45:117-124. doi 10.3233/NRE-192773.

10. Fan H, Tang H, Shan L, Liu S, Huang D, Chen X, Chen Z, Yang M, Yin X, Yang H, Hao D (2019) Quercetin prevents necroptosis of oligodendrocytes by inhibiting macrophages/microglia polarization to M1 phenotype after spinal cord injury in rats. J NEUROINFLAMM 16:1-15. doi 10.1186/s12974-019-1613-2.

11. Fehlings MG, Nakashima H, Nagoshi N, Chow DSL, Grossman RG, Kopjar B (2016) Rationale, design and critical end points for the Riluzole in Acute Spinal Cord Injury Study (RISCIS): a randomized, double-blinded, placebo-controlled parallel multi-center trial. SPINAL CORD 54:8-15. doi $10.1038 /$ sc.2015.95.

12. Gensel JC, Zhang B (2015) Macrophage activation and its role in repair and pathology after spinal cord injury. BRAIN RES 1619:1-11. doi 10.1016/j.brainres.2014.12.045.

13. Hall OT, McGrath RP, Peterson MD, Chadd EH, DeVivo MJ, Heinemann AW, Kalpakjian CZ (2019) The Burden of Traumatic Spinal Cord Injury in the United States: Disability-Adjusted Life Years. ARCH PHYS MED REHAB 100:95-100. doi 10.1016/j.apmr.2018.08.179.

14. James SLTA (2019) Global, regional, and national burden of neurological disorders, 1990-2016: a systematic analysis for the Global Burden of Disease Study 2016. Lancet Neurol. 18, 459-480. https://doi: 10.1016/S1474-4422(18)30499-X

15. Jiang K, Zhuang Y, Yan M, Chen H, Ge A, Sun L, Miao B (2016) Effects of riluzole on P2X7R expression in the spinal cord in rat model of neuropathic pain. NEUROSCI LETT 618:127-133. doi 10.1016/j.neulet.2016.02.065. 
16. Karadimas SK, Laliberte AM, Tetreault L, Chung YS, Arnold P, Foltz WD, Fehlings MG (2015) Riluzole blocks perioperative ischemia-reperfusion injury and enhances postdecompression outcomes in cervical spondylotic myelopathy. SCI TRANSL MED 7:194r-316r. doi 10.1126/scitransImed.aac6524.

17. Kigerl KA, Gensel JC, Ankeny DP, Alexander JK, Donnelly DJ, Popovich PG (2009) Identification of two distinct macrophage subsets with divergent effects causing either neurotoxicity or regeneration in the injured mouse spinal cord. J NEUROSCI 29:13435-13444. doi 10.1523/JNEUROSCI.325709.2009 .

18. Kobashi S, Terashima T, Katagi M, Nakae Y, Okano J, Suzuki Y, Urushitani M, Kojima H (2020) Transplantation of M2-Deviated Microglia Promotes Recovery of Motor Function after Spinal Cord Injury in Mice. MOL THER 28:254-265. doi 10.1016/j.ymthe.2019.09.004.

19. Lai H, Lu C, Wong C, Lin B, Chan S, Kuo C, Wu Z (2018) Baicalein attenuates neuropathic pain and improves sciatic nerve function recovery in rats with partial sciatic nerve transection. J CHIN MED ASSOC 81:955-963. doi 10.1016/j.jcma.2018.03.014.

20. Lee Y, Cho D, Kim CH, Han I, Gil EY, Kim K (2019) Effect of curcumin on the inflammatory reaction and functional recovery after spinal cord injury in a hyperglycemic rat model. The Spine Journal 19:2025-2039. doi 10.1016/j.spinee.2019.07.013.

21. Lin S, Xu C, Lin J, Hu H, Zhang C, Mei X (2021) Regulation of inflammatory cytokines for spinal cord injury recovery HISTOL HISTOPATHOL. 36, 137-142. https://doi: 10.14670/HH-18-262

22. Liu J, Wang $L$ (2018) The efficacy and safety of riluzole for neurodegenerative movement disorders: a systematic review with meta-analysis. DRUG DELIV 25:43-48. doi 10.1080/10717544.2017.1413446.

23. Liu Z, Ran Y, Huang S, Wen S, Zhang W, Liu X, Ji Z, Geng X, Ji X, Du H, Leak RK, Hu X (2017) Curcumin Protects against Ischemic Stroke by Titrating Microglia/Macrophage Polarization. FRONT AGING NEUROSCI 9:233. doi 10.3389/fnagi.2017.00233.

24. Mortazavi MM, Verma K, Harmon OA, Griessenauer CJ, Adeeb N, Theodore N, Tubbs RS (2015) The microanatomy of spinal cord injury: a review. CLIN ANAT 28:27-36. doi 10.1002/ca.22432.

25. Nagoshi N, Nakashima H, Fehlings MG (2015) Riluzole as a neuroprotective drug for spinal cord injury: from bench to bedside. MOLECULES 20:7775-7789. doi 10.3390/molecules20057775.

26. Oyinbo CA (2011) Secondary injury mechanisms in traumatic spinal cord injury: a nugget of this multiply cascade. Acta Neurobiol Exp (Wars) 71:281-299

27. Qin H, Holdbrooks AT, Liu Y, Reynolds SL, Yanagisawa LL, Benveniste EN (2012) SOCS3 deficiency promotes M1 macrophage polarization and inflammation. J IMMUNOL 189:3439-3448. doi 10.4049/jimmunol.1201168.

28. Rivlin AS, Tator $\mathrm{CH}$ (1977) Objective clinical assessment of motor function after experimental spinal cord injury in the rat. J NEUROSURG 47:577-581. doi 10.3171/jns.1977.47.4.0577.

29. Saijo K, Glass CK (2011) Microglial cell origin and phenotypes in health and disease. NAT REV IMMUNOL 11:775-787. doi 10.1038/nri3086. 
30. Wu Q, Zhang Y, Zhang Y, Zhang W, Zhang W, Liu Y, Xu S, Guan Y, Chen X (2020) Riluzole improves functional recovery after acute spinal cord injury in rats and may be associated with changes in spinal microglia/macrophages polarization. NEUROSCI LETT, 134829. doi: 10.1016/j.neulet.2020.134829

31. Xu J, He J, He H, Peng R, Xi J (2017) Comparison of RNAi NgR and NEP1-40 in Acting on Axonal Regeneration After Spinal Cord Injury in Rat Models. MOL NEUROBIOL 54, 8321-8331. https://doi: 10.1007/s12035-016-0315-3

32. Zeng H, Liu N, Yang YY, Xing HY, Liu XX, Li F, La GY, Huang MJ, Zhou MW (2019) Lentivirus-mediated downregulation of alpha-synuclein reduces neuroinflammation and promotes functional recovery in rats with spinal cord injury. J Neuroinflammation 16:283. doi 10.1186/s12974-019-1658-2.

33. Zhang Y, Liu Z, Zhang W, Wu Q, Zhang Y, Liu Y, Guan Y, Chen X (2019) Melatonin improves functional recovery in female rats after acute spinal cord injury by modulating polarization of spinal microglial/macrophages. J NEUROSCI RES 97:733-743. doi 10.1002/jnr.24409.

\section{Figures}


(a)

(b)

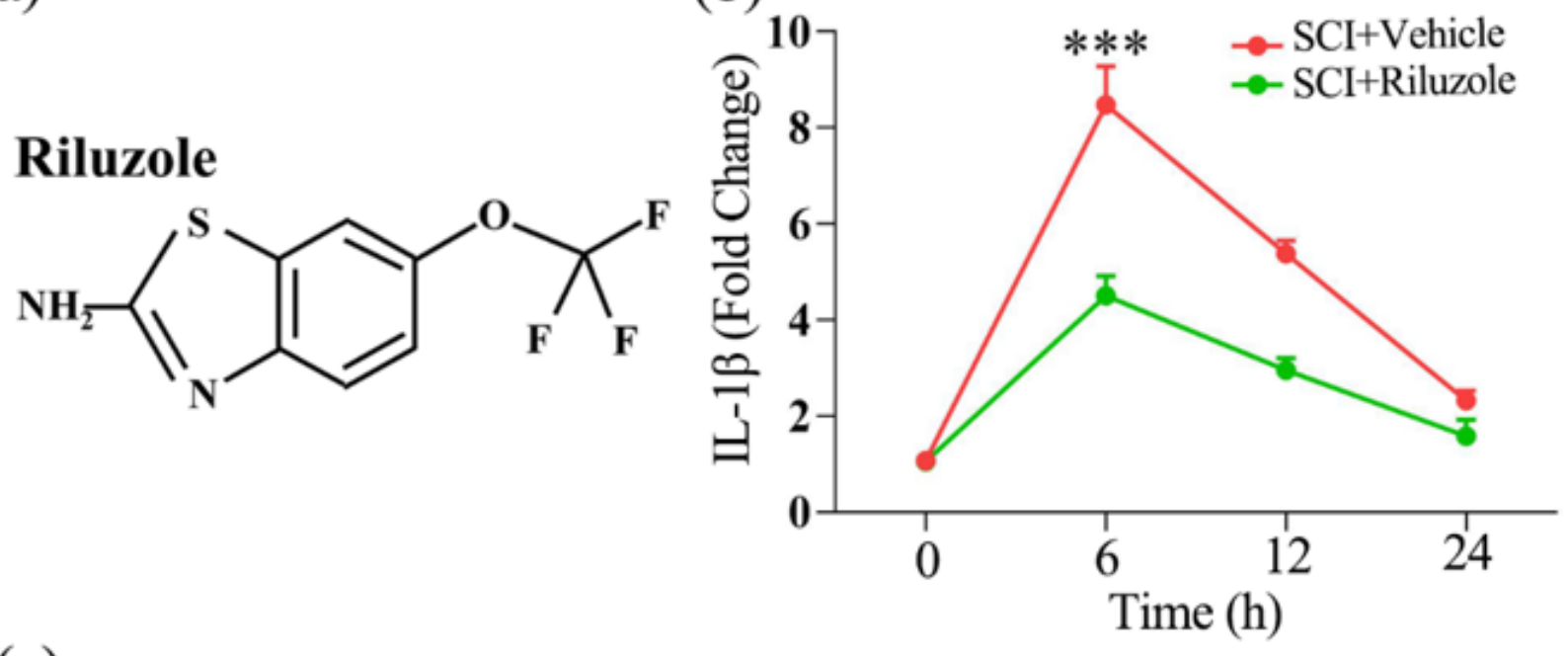

(c)

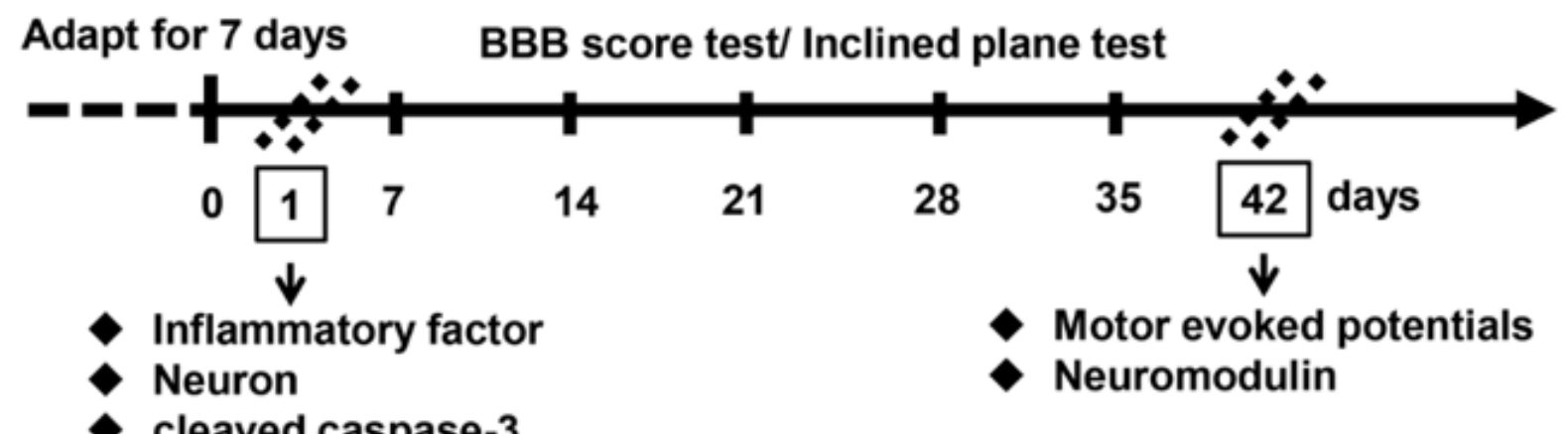

(d)
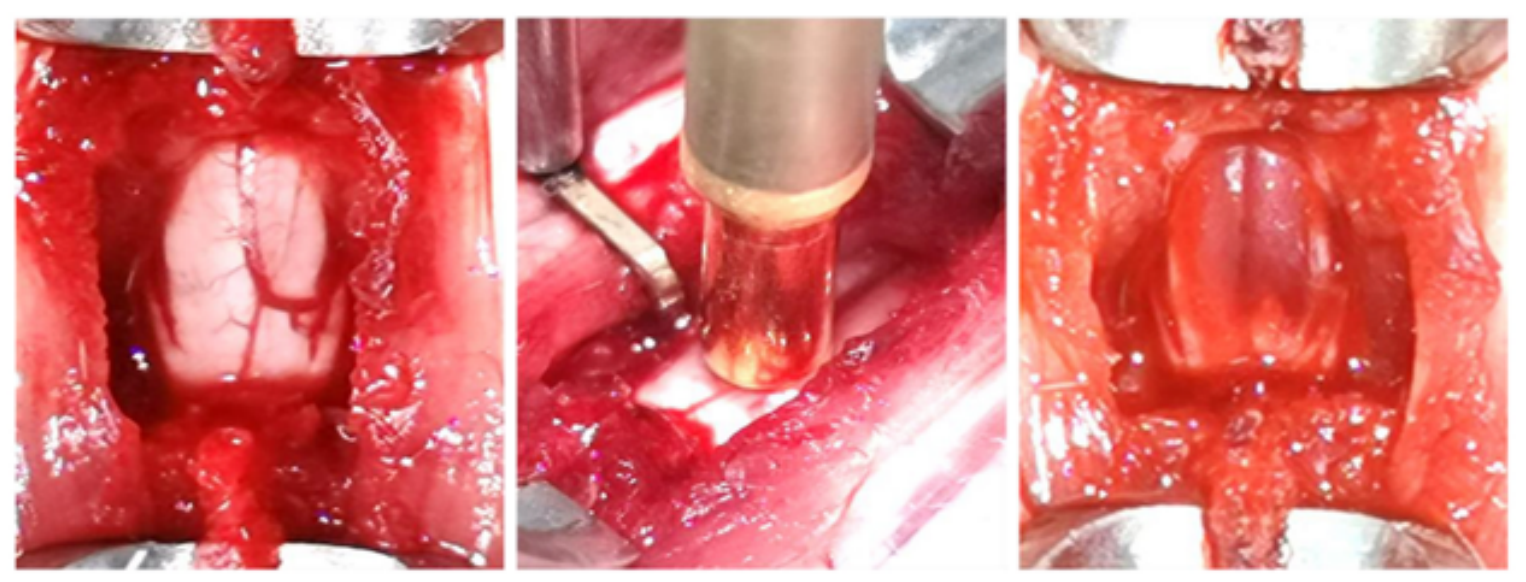

Figure 1

Riluzole reduces the release of the pro-inflammatory cytokine, IL-1 $\beta$, after SCl. (a) Molecular formula of riluzole. (b) A significant elevation of the mRNA level of IL-1 $\beta$ was noted $6 \mathrm{~h}$ after spinal cord injury (SCI). Riluzole applied in a single dose (6 mg/ $\mathrm{kg}$; intraperitoneally) immediately after the SCl decreased the mRNA level of IL-1 $\beta$ after $6 \mathrm{~h}$. Data are presented as the mean $\pm \mathrm{SEM}, \mathrm{n}=6$ per group, ${ }^{\star \star \star} \mathrm{P}<0.001: \mathrm{SCl}+$ 
Riluzole versus $\mathrm{SCl}+$ Vehicle, and two-way analysis of variance. (c) The experimental design. (d) The SCl surgical procedure.

(a)

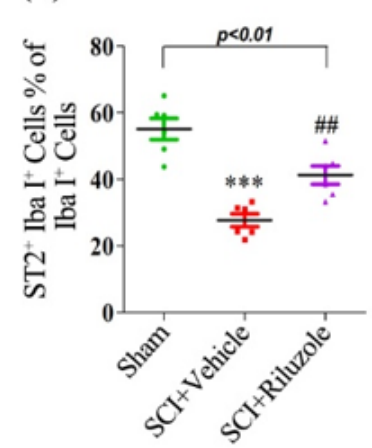

(b)

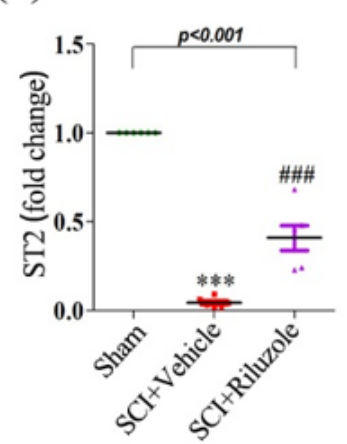

(c)

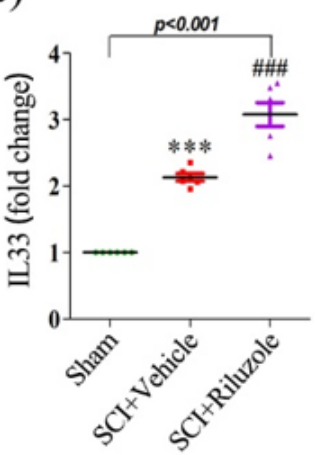

(d)

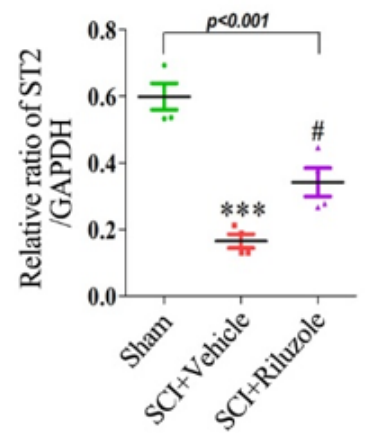

(e)

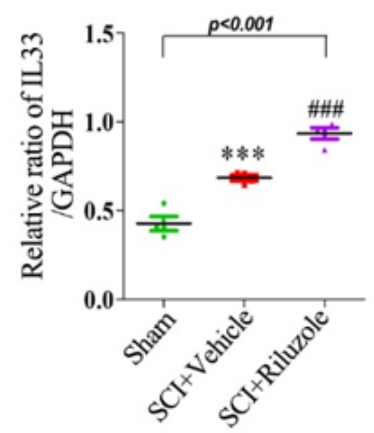

(f)

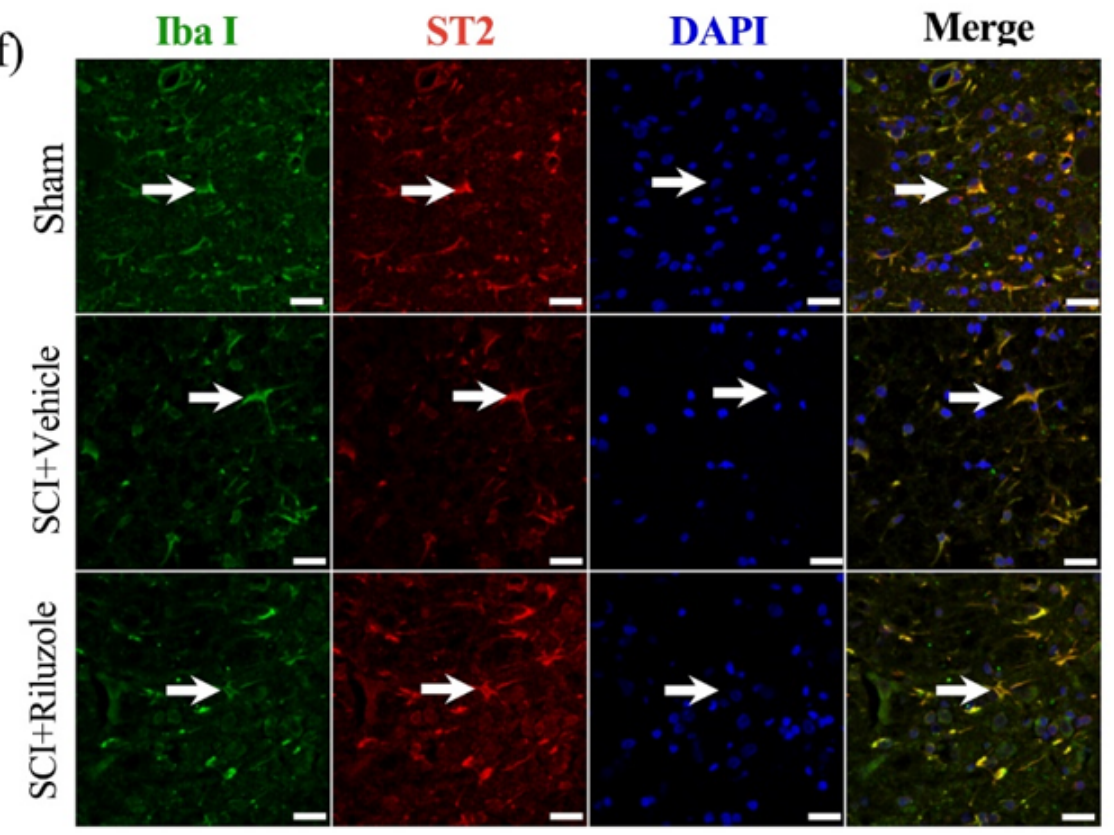

(g)

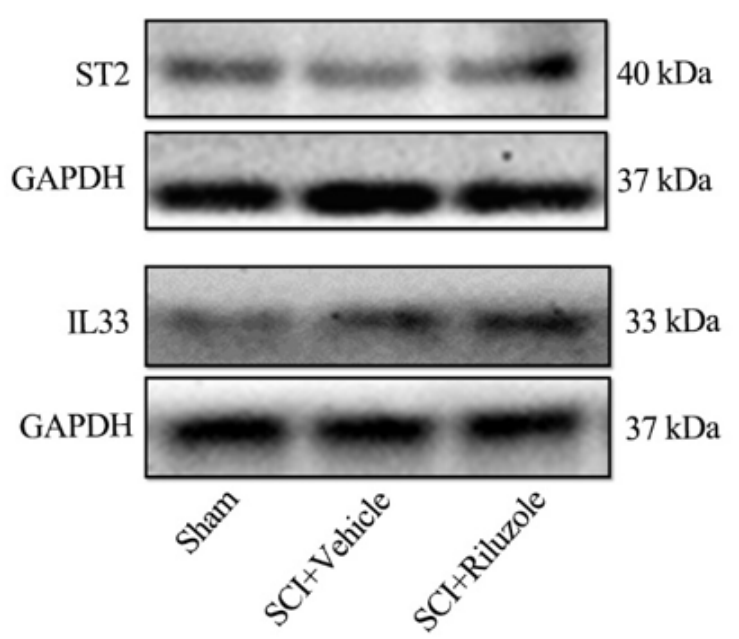

\section{Figure 2}

Riluzole increases the expressions of IL-33 and ST2 in microglia/macrophages after acute spinal cord injury (SCl). (a) Representative images show double-immunostaining of Iba I and ST2 at 1 day after SCI (scale bar: $50 \mu \mathrm{m}$ ). (b) Representative western blots show protein expressions of IL-33 and ST2 in each group at 1 day after SCl. (c) Quantification of Iba I+/ST2+ double-labeled cells (percentage of total Iba I+ cells) in each group. (d, e) The mRNA levels of ST2 and IL-33 in each group. (f, g) ST2 and IL-33 protein expression levels in each group. Data are presented as the mean $\pm S E M, n=6$ per group (one-way analysis of variance). ${ }^{*} * \mathrm{P}<0.001, \mathrm{SCl}+$ Vehicle versus Sham; $\# \mathrm{P}<0.05 ; \# \# \mathrm{P}<0.01 ; \# \# \# \mathrm{P}<0.001, \mathrm{SCl}$ + Riluzole versus $\mathrm{SCl}+$ Vehicle. 
(a)

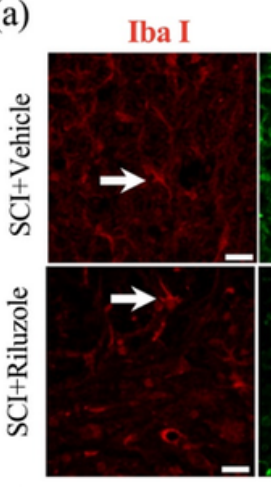

(c)

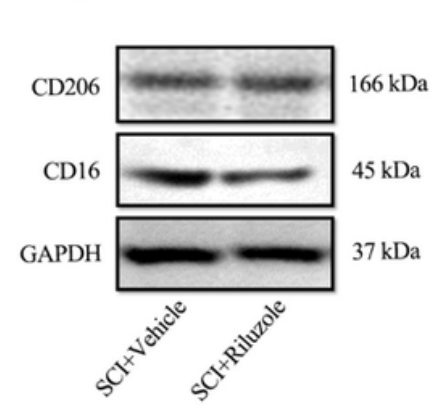

CD16

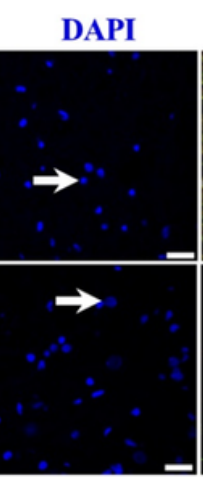

(d)

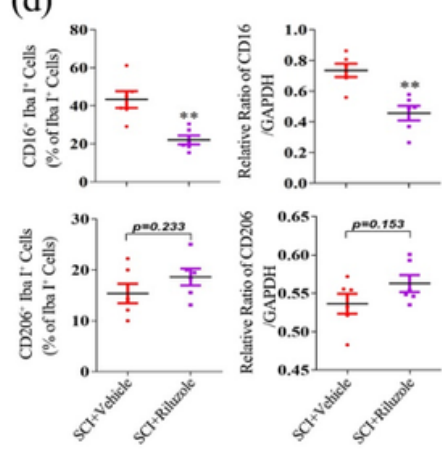

(b)

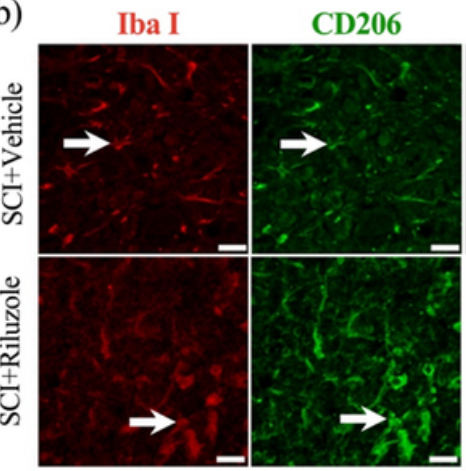

(e)
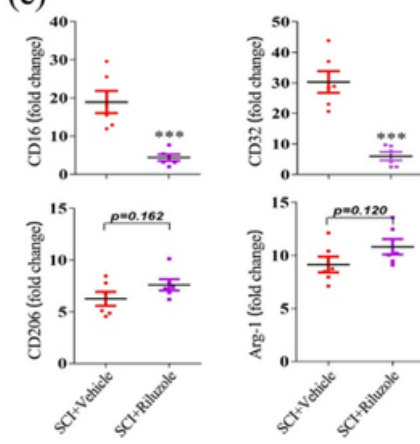

DAPI Merge
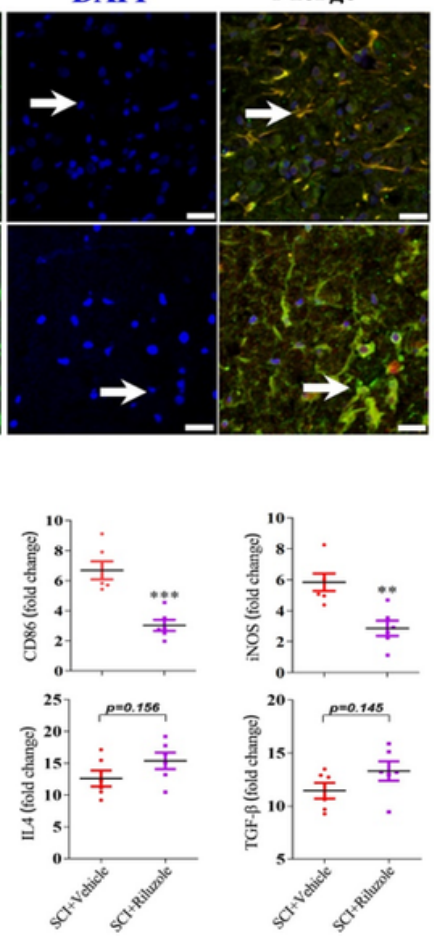

\section{Figure 3}

Riluzole inhibits microglia/macrophage M1 expression in spinal cord injury (SCI) rats. (a) Representative images show double-immunostaining of Iba I and CD16 in rats at 3 days after SCI (scale bar: $50 \mu \mathrm{m}$ ). (b) Double-immunostaining of Iba I and CD206 (scale bar: $50 \mu \mathrm{m}$ ). (c) Representative western blot shows expression of CD206 and CD16 at 3 days post-SCI. (d) Quantification of CD16+/Iba I+ and CD206+/Iba I+ double-labeled cells (percentage of total Iba I+ cells), and CD16 and CD206 protein levels in each group. Glyceraldehyde 3-phosphate dehydrogenase was used as the loading control. (e) The mRNA levels of CD16, CD32, CD86, iNOS, CD206, Arg-1, IL-4, and TGF- $\beta$ in each group. Data are presented as the mean \pm SEM, $\mathrm{n}=6$ per group. Student's t-test. ${ }^{\star *} \mathrm{P}<0.01$; ${ }^{* \star} \mathrm{P}<0.001, \mathrm{SCI}+$ Riluzole versus $\mathrm{SCI}+$ Vehicle. 
(a)

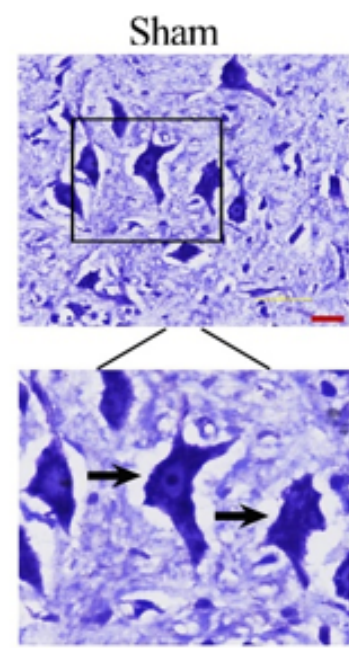

(b)
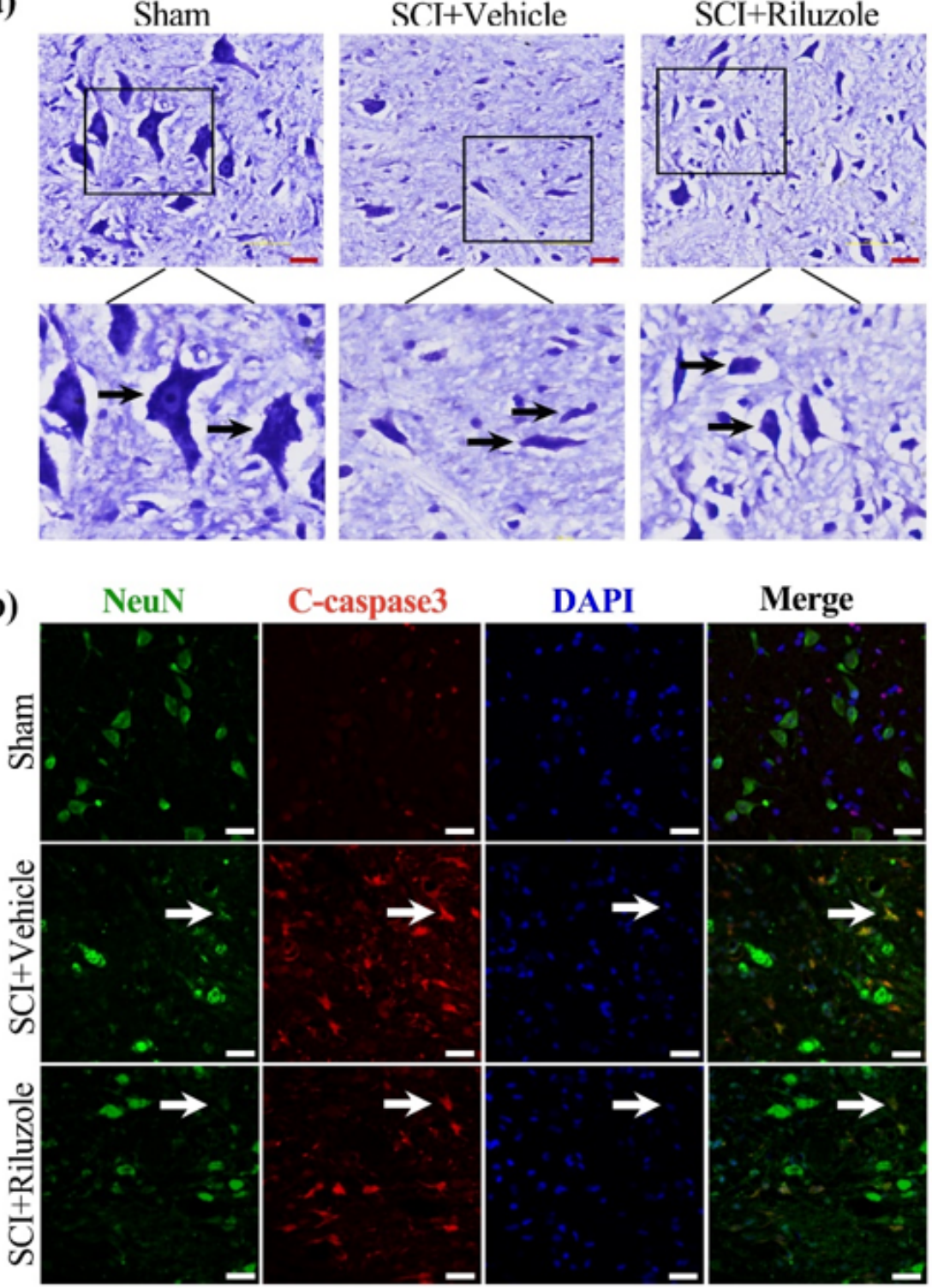

(c)

(d)
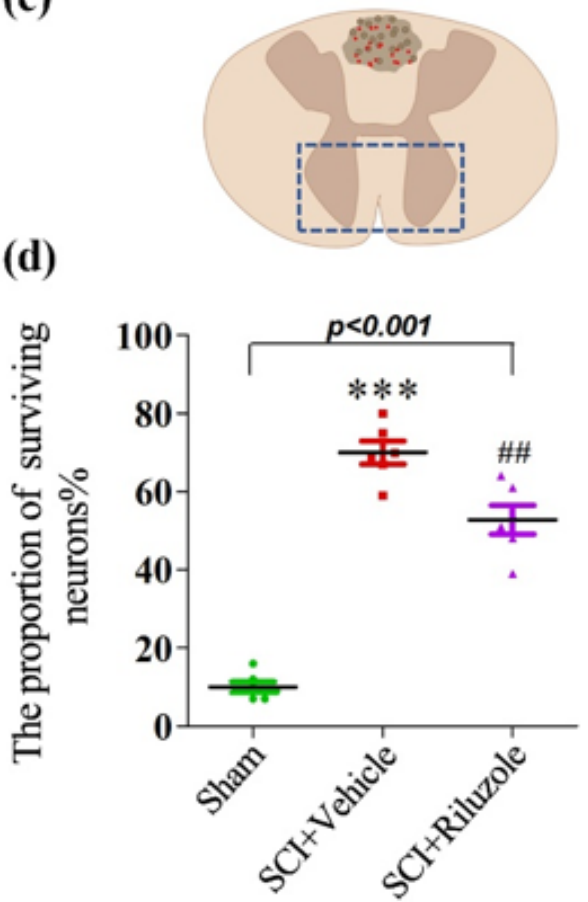

(e)

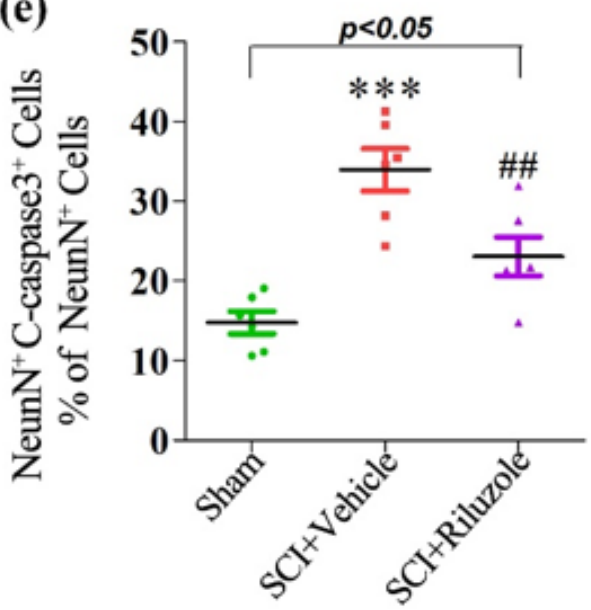

Figure 4

Riluzole reduces neuronal loss after acute spinal cord injury (SCl). (a) Representative Nissl staining of Sham, $\mathrm{SCl}+$ Vehicle, and SCl + Riluzole rats at 1 day after $\mathrm{SCl}$ (scale bar: $50 \mu \mathrm{m}$ ). (b) Representative images show double-immunostaining of cleaved-caspase- 3 and NeuN in rats at 1 day after SCl (scale bar: $50 \mu \mathrm{m}$ ). (c) In this diagram of the ventral horn, the boxed area shows the region used for counting neurons. (d) Quantification of the percentage of surviving neurons at 1 day post-SCl in each group. (e) Quantification of cleaved-caspase-3+/NeuN+ double-labeled cells (percentage of total NeuN+ cells) in each group. Data are presented as the mean $\pm S E M, n=6$ per group (one-way analysis of variance). ${ }^{*} * *$ $<0.001, \mathrm{SCl}+$ Vehicle versus Sham; \#\#P $<0.01, \mathrm{SCl}+$ Riluzole versus $\mathrm{SCl}+$ Vehicle. 
(a)

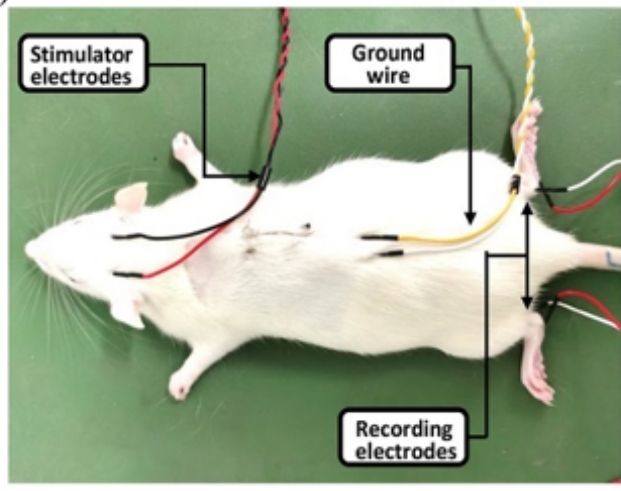

(d)

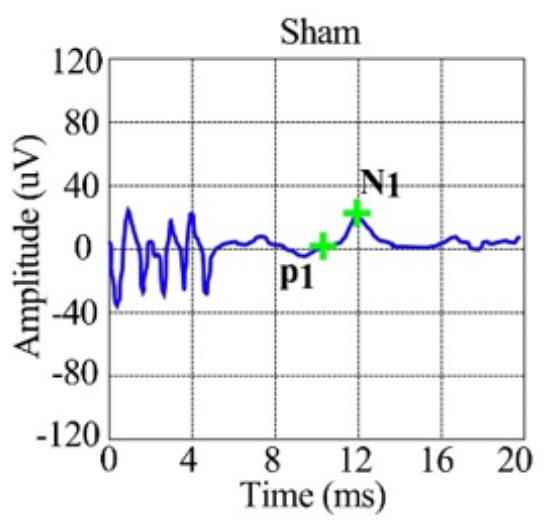

(g)
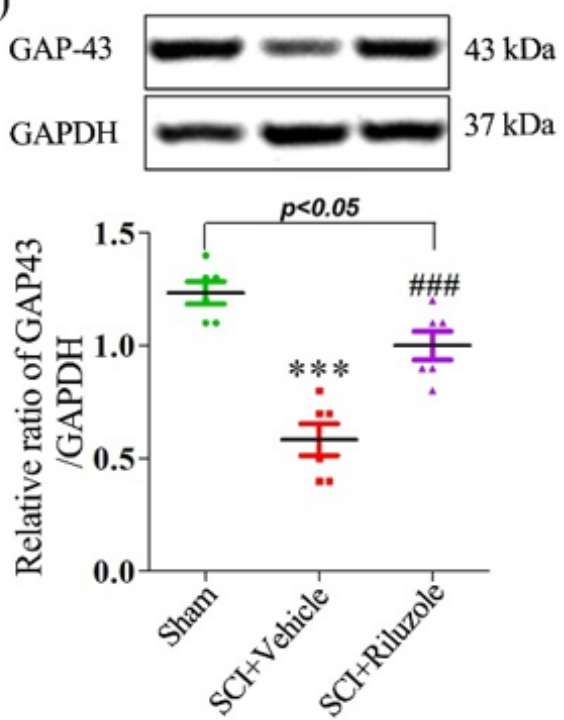

(b)

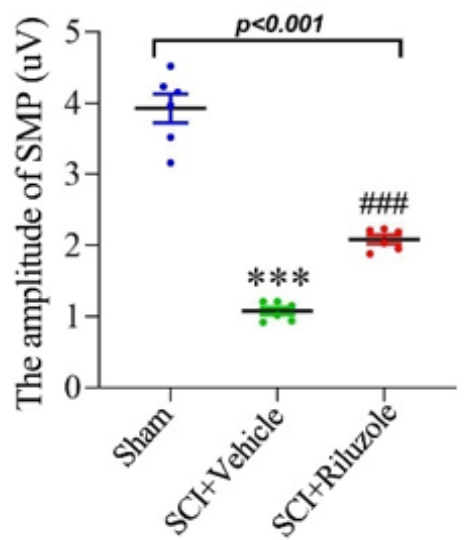

(e)

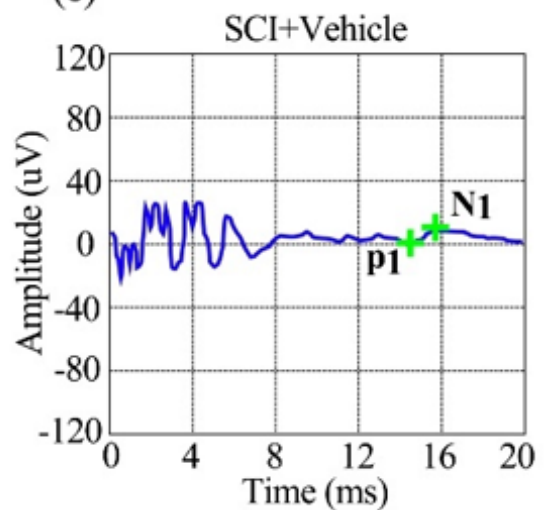

(h)

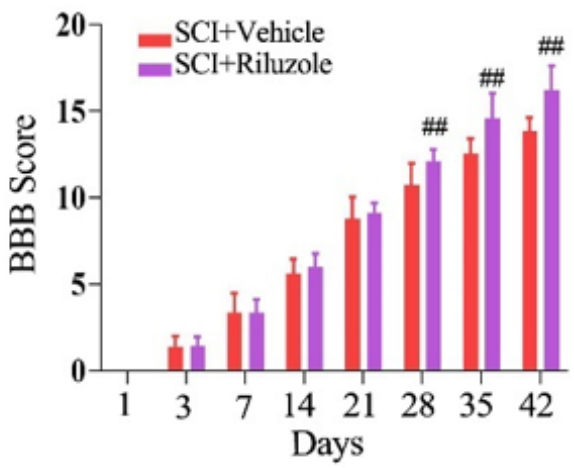

(c)

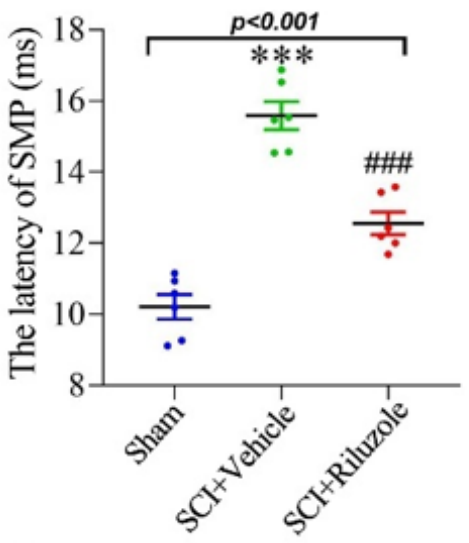

(f)

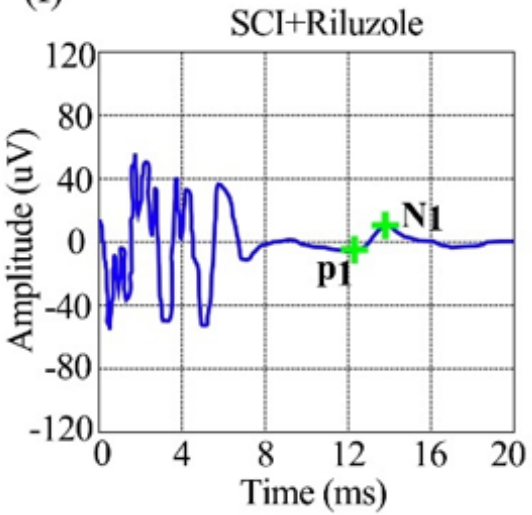

(i)

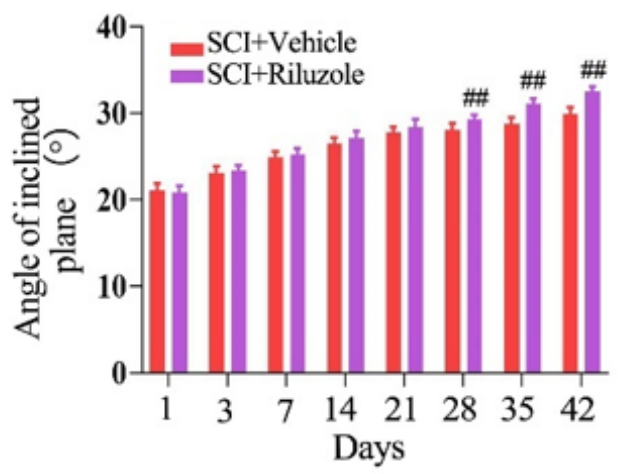

Figure 5

Riluzole promotes recovery of neurological function at 6 weeks post-spinal cord injury (SCl). (a) Schematic diagram of motor evoked potentials (one-way analysis of variance). (b-c) Quantification of the latency and amplitude of motor evoked potentials (MEPs). (d-f) Representative waveform of MEPS. (g) Western blot analysis of GAP43 protein expressions in each group (one-way analysis of variance). (h-i) Quantification of scores in the Basso, Beattie, Bresnahan test and inclined plane test from day 1 to week 
6 after $\mathrm{SCl}, \mathrm{n}=6$ per group (Student's t-test). Data are presented as the mean $\pm \mathrm{SEM}, \mathrm{n}=6$ per group. ${ }^{\star \star \star} \mathrm{P}$ $<0.001, \mathrm{SCl}+$ Vehicle versus Sham; \#\#P $<0.01$, \#\#\# $<0.001, \mathrm{SCl}+$ Riluzole versus $\mathrm{SCl}+$ Vehicle. 